Ennis, H. L. \& Sussman, M. (1958). J. gen. Microbiol. 18, 433-449

\title{
Synergistic Morphogenesis by Mixtures of Dictyostelium discoideum Wild-type and Aggregateless Mutants
}

\author{
By H. L. ENNIS AND M. SUSSMAN \\ Northwestern University, Evanston, Illinois, U.S.A.
}

SUMMARY: Aggregations involving mixtures of wild-type and 'aggregateless' mutant organisms of Dictyostelium discoideum were examined. The proportions of wild-type individuals which could initiate the formation of aggregative centres by their neighbours was found to depend markedly upon the particular mutant in the mixture. The mutants appear to have supplied some of the cells responding to the initiative stimulus where these were needed and also to have produced diffusible material enabling many more wild-type organisms to act as initiators than would ordinarily do so. The results also indicate the existence of a variety of cellular interactions which may underlie the normal morphogenetic sequence.

Aggregation of the myxamoebae of cellular slime moulds occurs during the stationary phase. The cells elongate and move in radial streams towards centres of aggregation. Subsequently each aggregate is transformed into a slug-shaped pseudoplasmodium and ultimately into a terminal fruiting body. One experimental approach to the aggregative process was made available by the finding that myxamoebae, washed free of their bacterial associates and dispensed on washed agar, did not proliferate but could aggregate in normal fashion ultimately to produce fruits with viable spores. Some of the salient facts obtained by studies along these lines (Sussman \& Noel, 1952; Sussman, 1952, 1955) follow.

(1) The number of aggregative centres produced by a population of constant size is a function of the population density. A maximum number of centres was formed at the optimal density. On the low side, progressively fewer centres appeared until at the threshold density none were formed due presumably to ineffectiveness of the chemotactic stimulus. On the high side, centres were larger but fewer, due presumably to competition between neighbouring potential centre-forming agencies. The precise values of the threshold and optimal densities varied with species and strain. (The performance of Dictyostelium discoideum wild-type is illustrated by the control curve in Fig. 6.)

(2) At the optimal density, the number of aggregative centres is directly proportional to the number of cells present. For Dictyostelium discoideum wildtype, the ratio of centres formed to cells present was 1:2200. Mutants and other species yielded different values.

(3) Not all cells in the population can initiate aggregative centres. In small replicate population samples, the ability to initiate a centre was distributed at random as a single event (i.e. statistically consistent with the Poisson series). Under these conditions the ratio of centres formed to cells present was again $1: 2200$. 
(4) Synergistic aggregations occur when Dictyostelium discoideum wildtype cells, at too low a density to aggregate alone, are mixed with 'aggregateless' mutant cells, incapable of aggregating spontaneously. Small constant numbers of wild-type myxamoebae were mixed with varying numbers of aggregateless cells. The number of centres increased progressively with the addition of mutant cells until, in the presence of excess aggregateless individuals, a plateau was reached. At the plateau, the number of centres formed was directly proportional to the number of wild-type in the mixture. When mixed with one aggregateless stock, the plateau ratio of centres formed to wild-type present was $1: 980$; with another mutant, $1: 1780$.

Consideration of the first three statements compelled us to accept the view that the formation of each aggregative centre requires the presence of a special cell in the population. This cell, termed the initiator, induces its neighbours, the responder cells, to begin aggregation. The fourth statement indicated that the number of cells which can act as initiators depends upon the kind of responder cell audience which they face. The present communication, concerned with a study of synergistic aggregations by Dictyostelium discoideum wild-type and 25 independently isolated aggregateless strains, gives a measure of the extent to which the ratio centre:wild-type cell can vary. The results also provide an insight into the way in which this change in the distribution of centres may be effected.

\section{METHODS}

Organisms and culture methods. Dictyostelium discoideum Raper, strain NC-4, was used. Some of the mutants arose spontaneously but the majority were obtained after u.v.-irradiation by procedures previously described (Sussman \& Sussman, 1953). All were isolated in pure culture by serial clonal passage, being grown upon glucose peptone agar (SM) in association with Aerobacter aerogenes (Sussman, 1951).

Aggregation experiments. Mass cultures on SM medium with Aerobacter aerogenes were incubated $40-44 \mathrm{hr}$. at $22^{\circ}$ by which time the bacteria had been ingested and the myxamoebae were in the stationary growth phase. The myxamoebae were harvested with cold distilled water and washed three times by centrifugation for $5 \mathrm{~min}$. at $1000 \mathrm{rev} . / \mathrm{min}$. in an International refrigerated centrifuge. The final pellet was suspended in salt solution (Bonner, 1947) and replicate direct counts were made in a Levy haemocytometer. Appropriate dilutions were dispensed in $\mathbf{0 . 0 1} \mathrm{ml}$. drops on washed agar in Petri dishes (Sussman, 1952). The Petri dishes were allowed to stand with covers ajar to permit absorption of the excess fluid by the agar and were incubated at $22^{\circ}$. Counts of aggregative centres were made under a dissecting microscope at the times indicated for the separate experiments.

Incubation on agar membranes. Thin agar membranes (30-60 $\mu$. thick) were prepared on stainless-steel membrane holders by a procedure described elsewhere (Sussman \& Lee, 1955) and incubated in sealed Stender dishes under a water-saturated atmosphere. Washed myxamoebae were dispensed by pipette on either or both sides. After permitting the cells to sink down on and adhere 
to the agar, most of the excess fluid was removed with a piece of filter paper. Incubation and counts of aggregative centres were conducted as previously described.

\section{RESULTS}

\section{Pedigrees and special properties of the aggregateless stocks}

Table 1 provides the pertinent data. As seen, three parental stocks were used: wild-type (NC-4), the bushy mutant (Bu-1), and the fruity mutant (Fty-1), themselves isolated from the wild-type after u.v.-treatment (Sussman \& Sussman, 1953; Sussman, 1955).

Table 1. Pedigree and special properties of aggregateless stocks of Dictyostelium discoideum

\begin{tabular}{|c|c|c|c|}
\hline Source & Strain number & Method of isolation & Special properties \\
\hline Wild-type & $\begin{array}{c}\mathbf{5 3}, \mathbf{5 3 - A}, \mathbf{5 9} \\
\mathbf{7 0 , 7 1 , 7 2} \\
73,74,78\end{array}$ & Ultraviolet irradiation & \multirow{4}{*}{$\begin{array}{l}\text { Sharply defined plaques with no } \\
\text { sign of aggregation when grown } \\
\text { on SM medium with Aerobacter } \\
\text { aerogenes. The myxamoebae are } \\
\text { morphologically indistinguish- } \\
\text { able from the wild-type }\end{array}$} \\
\hline & 87 & Spontaneous mutation & \\
\hline Mutant Bu-1 & $\begin{array}{l}204,206 \\
83,84\end{array}$ & $\begin{array}{l}\text { Ultraviolet irradiation } \\
\text { Spontaneous mutation }\end{array}$ & \\
\hline Mutant Fty-1 & 122 & Ultraviolet irradiation & \\
\hline Wild-type & $\begin{array}{l}75,76,77,79 \\
85,86,89 \\
91\end{array}$ & Ultraviolet irradiation & $\begin{array}{l}\text { Diffuse, rapidly spreading plaques } \\
\text { with no sign of aggregation } \\
\text { when grown on SM medium } \\
\text { with } A \text {. aerogenes. The myx- } \\
\text { amoebae are dwarfs. }\end{array}$ \\
\hline
\end{tabular}

The bushy mutant produces bushes of tiny fruiting bodies from each original aggregate. Washed myxamoebae on washed agar have a threshold density of 80 cells $/ \mathrm{mm}^{2}$ for any centre formation and an optimal density of 350 ; the respective values for the wild-type are 80 and 200 . At the optimal density the ratio centres:cells was $1: 1800$, scarcely different from that of the wildtype $(1: 2200)$. The fruity mutant is distinguished by the fact that it forms a multitude of tiny aggregates and a single correspondingly small but otherwise normal fruiting body from each. The threshold density for Fty-1 was found to be 40 and the optimal density, 200. The centre:cell ratio at the optimal density is $1: 24$.

When grown on SM agar medium with Aerobacter aerogenes, all of the aggregateless mutants produced plaques (colonies) devoid of any sign of aggregation. The specific plaque morphologies did differ as indicated in Table 1. In addition, one class of mutant stocks is distinguished by the fact that the myxamoebae are dwarfs. The mean diameter of such cells is $5 \mu$. in the stationary phase. Under the same conditions the mean diameters of wild-type or other aggregateless myxamoebae are all about $11 \mu$., in agreement with the figure arrived at by Bonner \& Frascella (1953).

Gregg (1957) showed that, of the dwarf strains, at least Agg-89 and Agg-91 differed antigenically from the wild-type. Good agglutination was obtained with homologous mixtures of myxamoebae and immune sera but no cross- 
reactions could be seen in heterologous mixtures. In contrast, the nondwarf aggregateless strains tested did not differ from the wild-type antigenically by this criterion.

\section{Synergistic aggregations}

Washed suspensions of wild-type and mutant myxamoebae were prepared and counted as described in the methods section. Mixtures were made and replicate $0.01 \mathrm{ml}$. drops were dispensed on washed agar. Further treatment and counts of aggregative centres were performed as previously described.

The mutant strains fell into four classes on the basis of their performances in this experiment. These will be described separately.

Mutants Agg-53, -53-A, -70, -77, -204 (class 1). The mutant cells when dispensed on washed agar failed to aggregate without exception. The wildtype, in the numbers used ( 2500 and $5000 / 0.01 \mathrm{ml}$. drop), produced either no or at most about one centre/drop. Because a $0.01 \mathrm{ml}$. drop covers $30 \mathrm{~mm} .^{2}$ of agar surface, the wild-type myxamoebae were at densities of 83 and 166 cells/ $\mathrm{mm} .^{2}$ respectively. The former is at the threshold for any centre formation at all. The latter permits only about $50 \%$ of the number of centres which normally would form at the optimal density of 200 cells $/ \mathrm{mm}^{2}$ (Sussman \& Noel, 1952). In the case of 5000 wild-type cells this would be $50 \%$ of $2 \cdot 3$ centres.

The mixtures of wild-type and mutant behaved as follows. The addition of aggregateless cells permitted a progressive increase in the number of centres until a plateau was attained. The plateau was conserved even with as many as $6 \times 10^{5}$ mutant cells/drop, a density of about $2 \times 10^{4}$ cells $/ \mathrm{mm} .^{2}$. Moreover, at the plateau, the number of centres was proportional to the number of wildtype present. Representative curves are shown in Figs. 1 and 2. Data relating to the constancy of the ratio centre:wild-type cell at the plateau are given in Table 2 for three of the mutants, and for the remaining two in Table 5.

Mutants Agg-85, -89, -91 (class 2). The synergistic performance of these strains is illustrated by the data given in Fig. 3. The numerical values for the ratio centre: wild-type cell at the plateau for Agg-91 are shown in Table 3. It may be seen that where small numbers of wild-type cells were used (500-1000), the curves were equivalent to those obtained with the mutants in class 1 above. That is, the number of centres formed at the plateau was proportional to the number of wild-type cells present and thus the ratio plateau content:wildtype cell remained constant at about 1:118. However, where higher numbers were used (2500-8000) the proportionality was not retained and the ratios progressively decreased. To account for this alteration, it must be noted that an increase in wild-type cells from 500 to 8000 within a drop of constant volume $(0.01 \mathrm{ml}$.) involves a 16 -fold increase in population density of the wildtype. An experiment was accordingly designed in which constant numbers of wild-type could be spread over a range of densities and yet still be in the presence of excess mutant cells. Replicate $0.01,0.03$, and $0.05 \mathrm{ml}$. drops were dispensed on washed agar. (The mean areas covered by these drops were 30, 66 , and $105 \mathrm{~mm} .^{2}$, respectively.) Each drop contained 8000 wild-type but the number of aggregateless cells varied with the size of the drop so as to provide a 


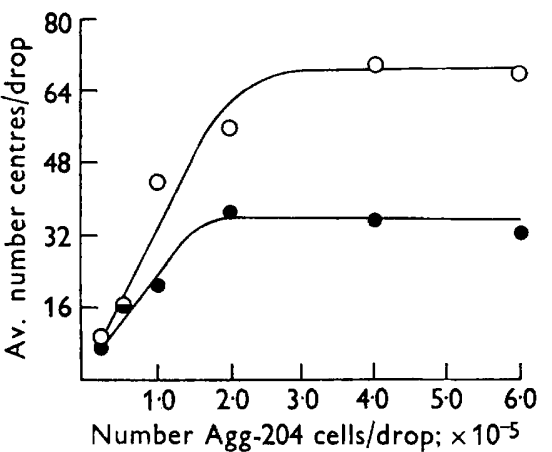

Fig. 1

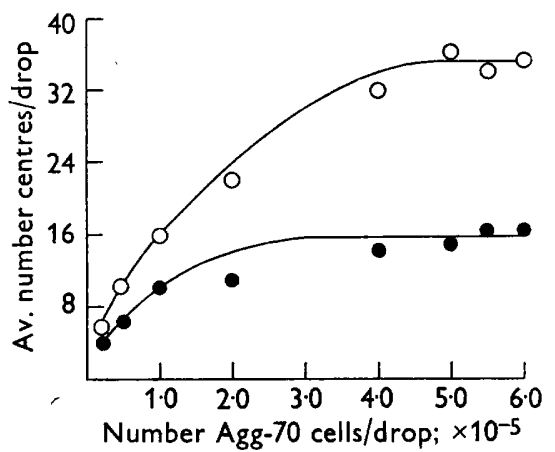

Fig. 2

Fig. 1. Mixtures of Dictyostelium discoideum wild-type and Agg-204. $O=5000$ wild-type cells/drop; $=2500$ wild-type cells/drop. Each point on the curves represents at least 32 separate determinations. Counts were made at 24 and $48 \mathrm{hr}$.

Fig. 2. Mixtures of Dictyostelium discoideum wild-type and Agg-70. $O=5000$ wild-type cells/drop; $=2500$ wild-type cells/drop. Each point on the curves represents at least 50 separate determinations. Counts were made at 24 and $48 \mathrm{hr}$.

Table 2. The ratios of centres formed to wild-type cells present when the latter were mixed with 3 aggregateless mutants of class 1

$\begin{array}{cccc}\begin{array}{c}\text { Aggregateless } \\ \text { strain employed }\end{array} & \begin{array}{c}\text { Number of wild- } \\ \text { type present }\end{array} & \begin{array}{c}\text { Mean number of } \\ \text { centres formed } \\ \text { at plateau }\end{array} & \begin{array}{c}\text { Ratio of } \\ \text { centres: wild-type } \\ \text { cells present }\end{array} \\ \text { Mutant Agg-70 } & 5000 & 35 & 1: 143 \\ & 2500 & 16 \cdot 3 & 1: 153 \\ \text { Mutant Agg-204 } & 5000 & 67 \cdot 8 & 1: 74 \\ & 2500 & 32 \cdot 2 & 1: 78 \\ \text { Mutant Agg-77 } & 5000 & 12 & 1: 417 \\ & 2500 & 6 \cdot 7 & 1: 373\end{array}$

Table 3. Ratios of centres to wild-type cells in mixtures of the latter with mutant Agg-91

Number of wildtype in mixture

500
750
1000
2500
5000
8000

Centre: wild-type cell ratio at plateau

$$
\begin{aligned}
& 1: 107 \\
& 1: 129 \\
& 1: 118 \\
& 1: 230 \\
& 1: 310 \\
& 1: 442
\end{aligned}
$$

constant density of $2 \times 10^{4}$ mutant cells $/ \mathrm{mm} .^{2}$, well above the value required to insure a plateau value for centre formation. Similar series of drops were dispensed containing 5000 and 2500 wild-type. The formation of centres by these mixtures is summarized in Table 4.

In the presence of excess aggregateless cells, 2500 wild-type cells at a density of 83 wild-type $/ \mathrm{mm}^{2}$ induced the formation of 10.9 centres. The same number at a density of 38 wild-type $/ \mathrm{mm} .{ }^{2}$ induced 13 ; at a density of 
Table 4. The dependence of centre formation on wild-type population density in synergistic mixtures of wild-type and mutant Agg-91

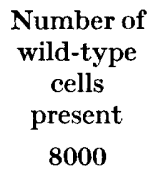

5000

2500

\section{in symergistic mixtures of wild-type and matant $A g-91$}

\begin{tabular}{|c|c|c|}
\hline \multicolumn{3}{|c|}{ Drop volumes (ml.) } \\
\hline 01 & 0.03 & 0.05 \\
\hline 33 & 121 & 76 \\
\hline 2 & $22 \cdot 5$ & 38 \\
\hline 442 & $1: 326$ & $1: 210$ \\
\hline 66 & 76 & 48 \\
\hline $6 \cdot 1$ & 19 & 27 \\
\hline$: 310$ & $1: 260$ & $1: 185$ \\
\hline & 38 & 24 \\
\hline & 13 & $18 \cdot 6$ \\
\hline $1: 230$ & $1: 192$ & $1: 134$ \\
\hline
\end{tabular}

24 wild-type $/ \mathrm{mm}^{2}{ }^{2}, 18 \cdot 6$ centres. It is therefore clear that by decreasing the wild-type density more centres were induced. In fact, at the lowest density, the ratio centre: wild-type cell was $1: 134$, bringing it within range of the ratios attained with 500-1000 wild-type, and thereby extending the proportionality between centres and wild-type cells. The data for 5000 and 8000 wild-type are consistent with this conclusion, although the plateau ratios still had not reached the value $1: 118$ at the lowest wild-type densities employed.
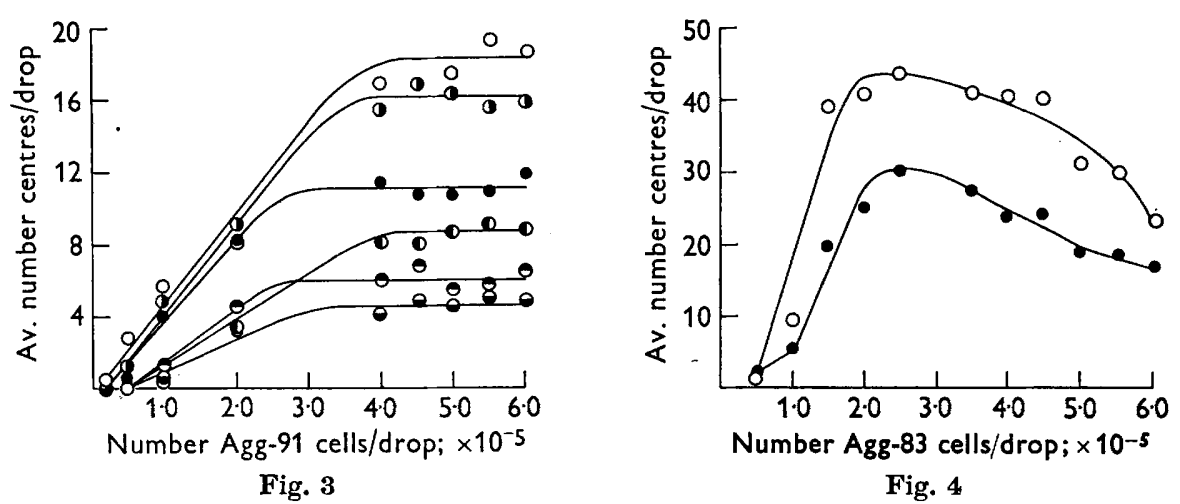

Fig. 3. Mixtures of Dictyostelium discoideum wild-type and Agg-91. $O=8000$ wild-type cells $/$ drop; $O=5000 ; \Theta=2500 ; O=1000 ; \theta=750 ; \theta=500$. Each point on the curve represents at least 50 separate determinations. Counts were made at 24 and $48 \mathrm{hr}$.

Fig. 4. Mixtures of Dictyostelium discoideum wild-type and Agg-83 showing an inhibition of aggregative centre formation at high concentrations of Agg-83. $O=5000$ wild-type cells/drop; $\mathbf{Q}=\mathbf{2 5 0 0}$ wild-type cells/drop. Each point on the curve represents at least 30 separate determinations.

The situation here encountered is seen to be somewhat analogous to that which obtains when wild-type myxamoebae serve as sole responders to their own initiators. In the latter circumstances, fewer centres are formed at densities above optimal $\left(200 \mathrm{cells} / \mathrm{mm} .^{2}\right)$ than at it. The difference between the two systems lies in the fact that though this interference also operates when small numbers of wild-type are faced with a saturation audience of mutant 
Agg-91, it does so at densities as low as $\mathbf{3 8}$ cells $/ \mathrm{mm}^{2}$. Clearly then, the presence of the mutant is required for the interference to operate at so low a density and it is fair to assume that the mutant exerts an interactive effect upon the wild-type to this end. But it must be recognized that this interference ultimately involves an interaction among the wild-type themselves since the diminution in centre formation becomes evident when the wild-type are brought closer to each other while yet maintaining a constant density of mutants.

Cellular interactions which mediate and control differentiation are commonly encountered in development systems (Grobstein, 1956) and in fact represent one of the problems basic to developmental biology. The above type of interaction is particularly interesting in that it involves the interaction of one cell class upon another which in turn causes the cells of class 2 to interact amongst themselves. The noteworthiness of these considerations stems from the fact that not even the ground rules of interactive relationships during development are known as yet, much less their biochemical mechanisms.

Mutants Agg-59, -71, -72, -73, -74, -78, -83, -84, -87, -206 (class 3). The synergistic capabilities of these mutants are complicated by the fact that when present in great numbers they inhibit centre formation. In some cases, the degree of inhibition is fairly slight as is illustrated in the results obtained by mixing wild-type with Agg-83 (Fig. 4). Here, centre formation rose to a maximum rather than a plateau and sharply declined with increasing numbers of mutant cells. Mixtures with Agg-74 and -206 gave similar results. In other cases, the degree of inhibition was more severe and the ability to form centres disappeared completely when the number of mutant cells exceeded a range of $5 \times 10^{4}$ to $2 \times 10^{5}$, depending on the particular strain used (Agg-59, $-71,-73,-78,-84)$. At the maxima, the numbers of centres were generally not proportional to the number of wild-type present but rather showed a steadily decreased slope. However, in a few strains proportionality was conserved. Two extreme cases of inhibition were observed (Agg-72, -87). No centres appeared in the mixtures no matter what the concentration of mutant cells. In fact, these mutants prevented centre formation even when the wild-type was present at a density high enough for it to aggregate alone.

Mutant Agg-121. A most remarkable performance was observed in mixtures of wild-type and this mutant; the data are summarized in Fig. 5. First, a double plateau was encountered. Attempts to show that this was due to the fact that the Agg-121 population contained two kinds of cells reacting differently to the presence of wild-type were uniformly unsuccessful. Secondly, the number of centres depended hardly at all upon the number of wild-type present within the range of $\mathbf{2 5 0 0 - 5 0 0 0}$ cells. This anomalous system is being further studied.

\section{The spectrum of centre: wild-type cell ratios}

Table 5 summarizes the best estimates of the ratio centre: wild-type cells. The figures without parentheses are firm estimates, based upon proportionate series of two or more plateau or maxima. The figures within parentheses were arrived 
at from non-proportionate series and represent the highest ratios observed in each series. The data further fortify the argument that the number of cells in the wild-type population which can act as initiators depends to a marked extent upon the nature of the audience which must be excited. As shown in Table 5 the most sensitive audience was mutant Agg-204, since they could

Table 5. The spectrum of centre:wild-type cell ratios permitted by the aggregateless mutants

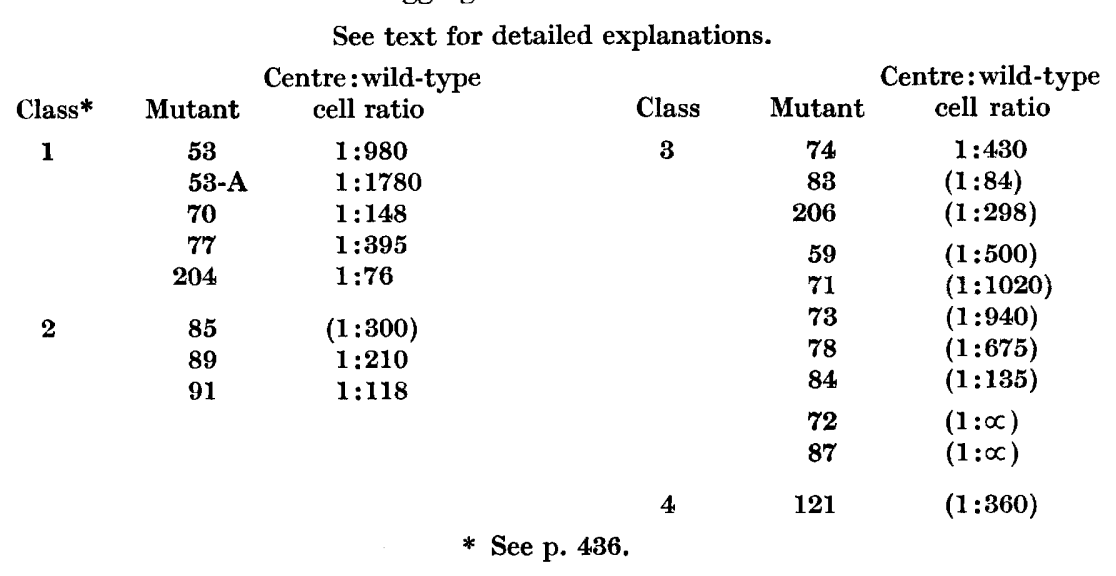

be induced to form centres by one out of 76 wild-type cells. However, it is clear that the response of any mutant population can not be taken simply as a reflexion of the efficiency of its sensing apparatus. Thus the performances of mutants Agg-72 and -87, were complicated by the inhibitory effect which they exerted upon the wild-type; in these cases no assessment of sensitivity is possible.

The synergistic relationship: the effect of wild-type upon mutants

The first question examined was: can the mutants aggregate under conditions where they are in close physiological contact with the wild-type but are physically separated? The point was tested by incubating washed cell suspensions on opposite sides of thin agar membranes. Agar membranes had been used (Sussman, 1954) in a study of synergistic fruiting by paired combinations of 13 morphogenetically deficient mutants including 6 aggregateless strains (five of which have been used in the present study) and 7 fruitless mutants which could aggregate but develop no further (i.e. unable to produce a pseudoplasmodium and terminal fruiting structure). When mixed in the proper cell ratios many paired combinations of these strains aggregated and produced terminal fruiting bodies but when incubated on opposite sides of agar membranes, they did not do so. Instead, they developed only to the extent that they did when alone. Further, when wild-type cells were opposed either by additional wild-type or by fruitless mutant cells across a membrane, the two populations aggregated in precise coincidence, each centre and radial cell stream above the membrane being reproduced in detail on the underside. 
Wild-type populations eventually produced pseudoplasmodia and subsequently terminal fruiting bodies while the fruitless populations stopped development after aggregating just as they normally do when alone. In contrast to the above, the aggregateless strains simply clustered immediately below wild-type centres and streams but remained unelongated and loosely packed. Pl. 1, fig. 1 shows a photomicrograph of this response. When the wildtype streams moved into their respective aggregates the 'pseudo-streams' remained in their original positions, the individual cells staying at the sites to which they had first been attracted. Only a few of the aggregateless strains had been tested in this fashion.

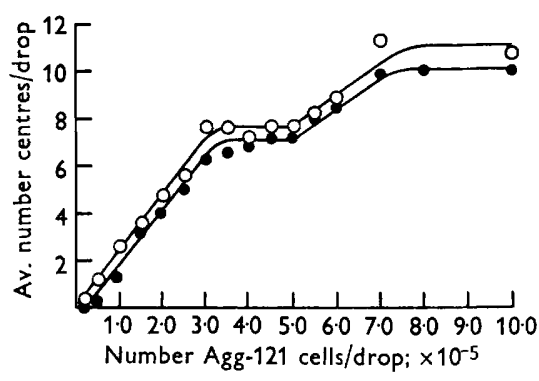

Fig. 5

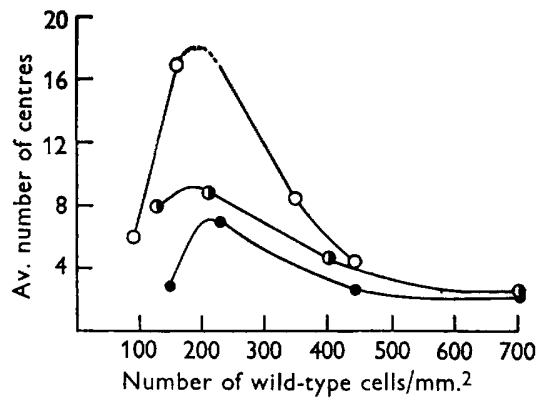

Fig. 6

Fig. 5. Mixtures of Dictyostelium discoideum wild-type and Agg-121, showing the double plateau. $O=5000$ wild-type cells/drop; $=2500$ wild-type cells/drop. Each point on the curve represents at least 100 separate determinations.

Fig. 6. Population density experiment, with 20,000 wild-type cells/drop, performed on a washed-agar substrate with the addition of an extract of mutant Agg-70 cells. $O=$ $240 \mathrm{ml}$. washed-agar $+60 \mathrm{ml}$. undiluted extract; $O=240 \mathrm{ml}$. washed-agar $+60 \mathrm{ml}$. $1 / 25$ dilution of extract; $O=240 \mathrm{ml}$. washed-agar $+60 \mathrm{ml}$. distilled water. Each point on the curve represents at least 20 separate determinations. Counts were made at 24 and $36 \mathrm{hr}$.

Since then the entire collection of 25 mutant stocks has been surveyed. No aggregateless mutant could produce a normal, complete aggregate when incubated across a membrane from the wild-type. In most cases they reacted precisely as described above. In a few cases, they remained completely unaffected and did not even cluster in coincidence with wild-type centres and streams. It would appear therefore that while at least some of the aggregateless cells can be chemotactically attracted at a distance by the wild-type, a separation of as little as $30 \mu$. (less than 3 cell diameters) can prevent the consummation of the synergistic relationship. Considering the present state of ignorance regarding the biochemical events which accompany aggregation and in particular the chemotactic process, it would be premature to offer a rationale for these observations.

The second question concerns the relative extents to which the mutant stocks did respond at a distance from the wild-type. One may ask, is there a correlation between the intensity with which a mutant reacts synergistically 
with wild-type in cell mixtures and the sensitivity with which it responds to the chemotactic apparatus of the wild-type when separated by a membrane? The relative responses of the $\mathbf{2 5}$ aggregateless stocks at a distance from the wild-type fell into three arbitrary classes, they were:

(a) Mutant cells did not respond at all to juxtaposed wild-type centres and streams but remained randomly distributed over the agar surface (mutants Agg-75, -76, -77, -79, -85, -86, -89, -91, -121, -126).

(b) The mutant cells clustered directly below wild-type centres but did not concentrate at the streams (mutants Agg-53, -59, -74, -83, -204, -206).

(c) The mutant cells clustered directly below wild-type centres and streams and more or less precisely duplicated the branchings of the latter. After the wild-type had completed its aggregation, the superimposed mutant streams remained as such or else were broken up and the cells were redistributed. The mutant cells did not elongate or pack closely even in the strongest reaction observed (mutants Agg-53-A, -70, -71, -72, -73, -78, -84, -87, -112).

Strains like mutant Agg-72 which formed no centres at all when mixed with wild-type were attracted very strongly across the $30 \mu$. of agar membrane. In contrast, the dwarf strains like mutants Agg-77 and -89 which readily formed centres when mixed with wild-type did not react at all when separated from them. Finally, whereas only one out of 1780 wild-type would induce Agg-53A cells to aggregate when mixed, one out of 76 wild-type were able to do so when mixed with Agg-204. Yet Agg-53A reacted more intensely than Agg-204 to the wild-type when separated by a membrane. Thus it is again obvious that the total synergistic performances of the mutant stocks can not be explained consistently in terms of a single parameter, in this case the chemotactic apparatus.

\section{The synergistic relationship: effect of mutants upon wild-type}

The data given here and previously (Sussman, 1952) reveal that the aggregateless mutants can affect centre formation in mixtures with wild-type under two entirely different circumstances. The first of these involves mixtures which contain relatively small numbers of wild-type (500-5000), too sparse by themselves to form any centres at all or, at most, too far below the optimal density to form more than a fraction of the centres of which they are otherwise capable. The addition of mutant cells not only permitted centres to form but led to the appearance of many more aggregates than the wild-type population itself could have produced at any density. The second circumstance involves mixtures containing relatively large numbers of wild-type $(6000-10,000)$ which by themselves would be at a density equal to or greater than optimal and so could form aggregates perfectly well without assistance from added mutant cells. Yet the addition of mutants led again to the appearance of many more centres than the wild-type could alone have produced. Further, when at least two of the mutants (Agg-53 and -53A) were present in excess, the ratios of centres : wild-type cells remained constant regardless of whether small or large numbers of wild-type took part in the synergistic aggregation (Sussman, 1952).

The mutants' ability to exert these two kinds of effect when physically separated from the wild-type was examined by paired incubation on thin 
agar membranes. A $0.01 \mathrm{ml}$. drop containing either 2000 wild-type (density $=67$ cells $/ \mathrm{mm} .{ }^{2}$ ) or 20,000 (density $=670$ cells $/ \mathrm{mm} .{ }^{2}$ ) was dispensed on one side of a washed-agar membrane and, after the excess fluid had been absorbed, a second drop of volume $0.03 \mathrm{ml}$. containing $3 \times 10^{5}$ mutant cells was dispensed on the other side. Control membranes bearing wild-type cells only were also prepared. Counts of aggregative centres produced by the wildtype were made at $24 \mathrm{hr}$. The drops containing 2000 wild-type produced no centres whatever, whether in the presence or absence of mutant cells on the other side of each membrane. Thus, under conditions where the wild-type is too sparse to aggregate alone, the mutant cells must actively take part if aggregation is to occur and the supply of diffusible materials is not sufficient for this purpose.

Table 6. The ability of aggregateless cells to stimulate centre formation by wild-type when separated by an agar membrane

See text for experimental details.

\begin{tabular}{|c|c|c|c|c|}
\hline \multirow[b]{2}{*}{ Mutant } & \multirow{2}{*}{$\begin{array}{c}\text { Ratio of } \\
\text { centres : wild-type } \\
\text { cells observed in } \\
\text { mixtures }\end{array}$} & \multicolumn{3}{|c|}{$\begin{array}{l}\text { Number of centres formed by } \\
20,000 \text { wild-type cells on agar } \\
\text { membranes }\end{array}$} \\
\hline & & $\begin{array}{l}\text { Experi- } \\
\text { mental }\end{array}$ & Control & $\begin{array}{c}\text { Fold } \\
\text { increase }\end{array}$ \\
\hline Agg-204 & $1: 76$ & $34 \cdot 2$ & $\mathbf{3} \cdot \mathbf{6}$ & $9 \cdot 5$ \\
\hline Agg-70 $\left(3 \times 10^{5}\right.$ cells $/$ drop $)$ & $1: 148$ & $30 \cdot 1$ & $3 \cdot 5$ & $8 \cdot 6$ \\
\hline Agg-70 $\left(1.5 \times 10^{5}\right.$ cells $/$ drop $)$ & $1: 148$ & $19 \cdot 1$ & $3 \cdot 5$ & $5 \cdot 4$ \\
\hline Agg-53 & $1: 980$ & $5 \cdot 3$ & $\mathbf{3 \cdot 2}$ & $1 \cdot 7$ \\
\hline Agg-72 & $1: \infty$ & $13 \cdot 8$ & - & $3 \cdot 8$ \\
\hline
\end{tabular}

The drops containing 20,000 wild-type gave the quite different results shown in Table 6. Three noteworthy facts are apparent. First, under conditions where the wild-type could aggregate unassisted, the passage of diffusible material from the mutants permitted the wild-type itself to form a multiplicity of aggregates. Secondly, the intensity of this effect depended on the number of mutant cells present, at least in the case of mutant Agg-70. Thirdly, the intensity of the effect was strain specific. For mutants Agg-53, -70 , and -204 , the capacity to enhance centre formation at a distance stood in the same relation as their respective abilities to do so when mixed with wildtype. Mutant Agg-72 is anomalous in that, while it actually inhibited centre formation when mixed with wild-type, it stimulated centre formation at a distance from the latter.

To summarize, the foregoing indicates that the aggregateless cells perform at least two functions in the synergistic mixtures: $(a)$ they take an active part in the aggregation as responder cells and can offset the lack of wild-type responders in doing so. This function is necessary only where a lack of responder cells exists; $(b)$ they produce diffusible material affecting the wild-type cells in such a way as to lead to a multiplicity of centres. When the number of wild-type in the mixture is small, both functions are performed by the mutants. When the number of wild-type is large, only the latter function (b) is necessary to produce the synergistic result. 
The effect of cell-free extracts on centre formation by the wild-type

The results of the last described membrane experiments raised the question of whether cell-free preparations from mutant stocks might not be able to affect wild-type aggregation in similar fashion. Preliminary results indicate that they do and further, that active preparations can be obtained not only from aggregateless cells but also from the fruity mutant Fty-1, and from the wild-type itself. To prepare extracts, the cells were harvested at the beginning of the stationary phase and ground in a mortar with powdered dry ice. The

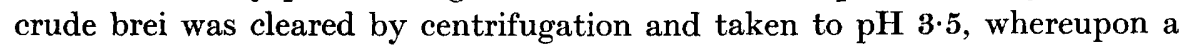
heavy proteinaceous precipitate settled out. The precipitate (inactive upon assay) was discarded; the supernatant fluid was neutralized and mixed with a suitable amount of molten washed agar and plates poured therefrom. Streptomycin sulphate $(0 \cdot 2 \%, \mathrm{w} / \mathrm{v})$ was included to prevent growth of bacteria and, in consequence, of the myxamoebae (Sussman, 1955).

Table 7. The effect of cell-free extracts on centre formation by

Dictyostelium discoideum wild-type

$\begin{array}{lc}\text { Source of extract } & \text { Activity* } \\ \text { Mutant Agg-70 } & \mathbf{2 \cdot 6} \\ \text { Mutant Agg-72 } & 2 \cdot 5 \\ \text { Mutant Fty-1 } & 11 \cdot 4 \\ \text { Wild-type } & 7 \cdot 8\end{array}$

* Ratio of the number of centres produced by 20,000 wild-type cells on extract-agar to number produced on washed-agar.

Bioassays were performed as follows. Appropriate dilutions of washed wild-type cells in salt solution were dispensed in drops on extract and control agars. The drops contained 20,000 cells over a range of population densities. The densities were determined by direct count with an ocular grid and were also calculated from measurements of the areas covered by the drops. Fig. 6 shows one assay of an extract obtained from mutant Agg-70. The extract did not affect the threshold or optimal density values nor did it alter the depression in number of centres encountered at densities above optimal. However, significantly greater number of centres appeared. At the optimal density the increase over the control value was $2 \cdot 6$-fold. Figure 7 shows the assay of a much more potent preparation from mutant Fty-1. An 11.4-fold increase in number of centres occurred at the optimal density. Table 7 contains a summary of the best results obtained with extracts from four strains of Dictyostelium discoideum. It is impossible to compare the relative potencies of these strains because replicate preparations have shown great fluctuation in activity. For one thing, the age of the culture at harvesting is extremely critical. Log phase cells provided inactive extracts. Cells considerably past the beginning of the stationary phase were much less active. In addition, the activity proved to be temperature-sensitive and degenerated upon storage, even in the cold. Notwithstanding these difficulties it may be said that preparations from mutant Fty-1 and wild-type were generally more active than those from 
the aggregateless strains although overlap was encountered. Previous studies (Bradley, Sussman \& Ennis, 1956; Sussman, 1956) showed that the initiative capacity of the wild-type is immune to a wide variety of external influences both chemical and physical. Thus the effects described above are at present unique and therefore represent a promising opportunity to examine the biochemical mechanisms underlying the initiation of aggregative centres.

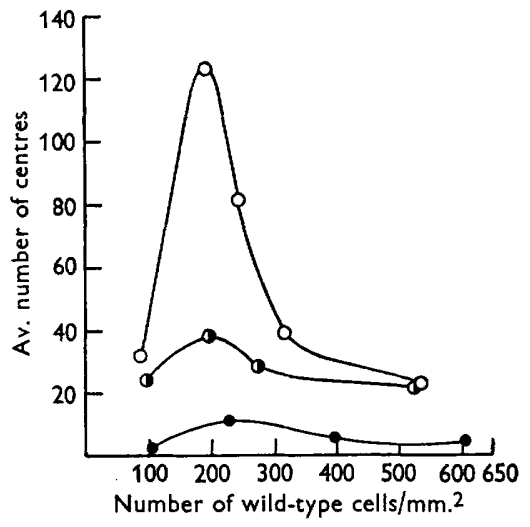

Fig. 7

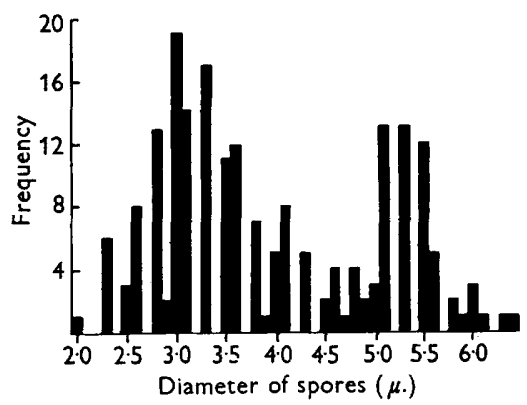

Fig. 8

Fig. 7. Population density experiment, with 20,000 wild-type cells/drop, performed on a washed-agar substrate with the addition of an extract of mutant Fty-1 cells. $O=240 \mathrm{ml}$. washed-agar $+60 \mathrm{ml}$. extract. $(=240 \mathrm{ml}$. washed-agar $+60 \mathrm{ml}$. $1 / 5$ dilution of extract. $0=240 \mathrm{ml}$. washed-agar $+60 \mathrm{ml}$. water. Each point on the curve represents 4 separate determinations.

Fig. 8. Size distribution of spores from fruiting bodies obtained by mixing 500 wild-type cells with $6 \times 10^{5} \mathrm{Agg-91}$ cells in a $0.01 \mathrm{ml}$. drop on a washed-agar medium. The size is given as the mean of the major and minor diameters. Two hundred spores were measured.

\section{The entrance of mutant cells into synergistic aggregates}

That cells of at least some aggregateless strains actually do enter the synergistic aggregates has been demonstrated in two ways. Spores from the resulting fruiting bodies were plated in clonal isolation to yield appreciable proportions of aggregateless clones, the precise values depending upon the nature of the paired strains and the proportions of myxamoebae originally mixed (Sussman, 1954). R. R. Sussman (unpublished data) vitally stained aggregateless cells and mixed them with wild-type. She found the stained cells in all parts of the fruiting structures.

The experiments to be described involved mixtures of wild-type and mutant Agg-91, and the results indicated that Agg-91 entered the aggregate and gave rise to dwarf spores which were incapable of germinating under conditions which permit wild-type spores to do so. Drops containing 500 wild-type and $6 \times 10^{5}$ cells of mutant Agg-91 were dispensed on washed-agar and allowed to fruit. Spore masses were suspended in water and examined in wet mounts. Two kinds of spores were evident: those resembling wild-type, and dwarf spores. Pl. 2 illustrates the size differences between these spores and between 
the corresponding vegetative cells. Measurements were made of the major and minor diameters of a few hundred individuals in the mixed spore population. The histogram Fig. 8 shows a bimodal distribution, one set of spores with a mean diameter of about $5 \cdot 4 \mu$. and the other set, about $3 \cdot 0 \mu$; the former value is characteristic of wild-type spores (Bonner \& Frascella, 1953).

Table 8. Efficiency of plating* and \% germination of spores from mixtures of Dictyostelium discoideum wild-type and Agg-91

\begin{tabular}{|c|c|c|c|c|c|c|}
\hline $\begin{array}{c}\text { Number of wild-cells } \\
\text { per drop }\end{array}$ & $\begin{array}{l}\text { Number of } \\
\text { spores } \\
\text { plated }\end{array}$ & $\begin{array}{l}\text { Plaque } \\
\text { count }\end{array}$ & EOP* & $\begin{array}{l}\text { Initial } \\
\text { spore } \\
\text { count }\end{array}$ & $\begin{array}{c}\text { Number of } \\
\text { non-germi- } \\
\text { nated spores } \\
\text { after } \\
\mathbf{2 4} \mathrm{hr} .\end{array}$ & $\begin{array}{l}\% \text { germi- } \\
\text { tion of } \\
\text { spores }\end{array}$ \\
\hline 5000 & - & - & - & 2008 & 188 & $90 \cdot 6$ \\
\hline 1000 & $\begin{array}{l}1595 \\
4149\end{array}$ & $\begin{array}{r}588 \\
1923\end{array}$ & $\left.\begin{array}{l}0 \cdot 37 \\
0 \cdot 46\end{array}\right\}$ & 3476 & 1341 & $61 \cdot 4$ \\
\hline 500 & 1575 & 290 & $0 \cdot 18$ & - & -- & - \\
\hline NC-4 alone & 1591 & 1290 & 0.81 & 1194 & 26 & $97 \cdot 8$ \\
\hline
\end{tabular}

Spores samples obtained from mixtures of $6 \times 10^{5}$ cells of mutant Agg-91 and different numbers of wild-type were plated out on SM agar with Aerobacter aerogenes. Among thousands of clones which appeared, only wild-type was encountered. However, the plating efficiency was positively correlated with the relative proportion of wild-type myxamoebae in the mixture (Table 8 ). The spores were also dispensed on SM agar without bacteria to determine $\%$ germination, the same correlation was encountered. In addition, the ungerminated spores were almost exclusively of the dwarf type.

The conclusion therefore appears inescapable that Agg-91 cells gave rise to the non-viable dwarf spores. That being so, the efficiency of plating and $\%$ germination values would seem to be reliable measures of the number of mutant cells which enter a synergistic aggregate (the spores make up $85 \%$ of the cellular assembly in a fruit, Bonner \& Slifkin, 1949; Sussman, 1955). Table 8 indicates that the mutant cells were a progressively decreasing part of the aggregates as the number of wild-type in the mixture increased. In drops containing more than 5000 wild-type the cellular contribution of the mutant would appear to be negligible. This is consistent with the conclusion derived from the agar membrane studies, namely that, where the number of wildtype is sufficient to permit them to aggregate alone, the synergistic contributions of the mutants need only to be in the form of diffusible material.

\section{Mixtures of aggregateless strains}

The elegant mutational analyses of Neurospora crassa, Escherichia coli, and other organisms have shown that simple metabolic lesions can account for the inability of trophically deficient mutants to grow under prescribed conditions. It seemed reasonable therefore to suppose that simple blockages 
of biosynthetic pathways might account for the inability of at least some morphogenetically deficient mutants of Dictyostelium discoideum to develop normally under defined conditions. With $E$. coli mutants Davis (1950) showed that strains possessing different lesions could mutually satisfy their deficiencies and thus grow synergistically on minimal medium. One might equally well expect that two different aggregateless stocks of $D$. discoideum would in the same way be capable of synergistic aggregation. A previous study tested this possibility with paired and trebled combinations of six independently derived aggregateless mutants which had shown clear phenotypic differences (Sussman, 1954); no synergistic aggregations were then observed. This survey has now been extended to 25 mutants. Of the 300 possible pairings, 217 were examined on washed-agar without success. Three different numbers of cells of each strain were mixed in all permutations. In addition, 79 pairs were grown together on SM agar with Aerobacter aerogenes. These produced no aggregates; neither did mixtures on washed-agar in which the positions of the partners were assymetrical It should be noted that syntrophy between $E$. coli mutants as described by Davis requires that there be accumulation of intermediates which occur before the metabolic lesion and that these be excreted in appreciable amounts. It is possible that these requirements are not met by the aggregateless strains of $D$. discoideum used here. On the other hand perhaps morphogenetic assemblies require for their proper functioning a delicately balanced matrix of interactions correctly oriented in space and time and that this is impossible to duplicate simply by mixing two unbalanced mutants.

\section{DISCUSSION}

The synergistic performance of Dictyostelium discoideum wild-type paired with eight of the aggregateless strains has provided evidence supporting the thesis that single wild-type cells are specially endowed to initiate aggregations: in the presence of excess mutant cells of these eight varieties (Agg-53, -53A, $-70,-74,-77,-89,-91,-204)$, the number of centres formed was linearly proportional to the number of wild-type present. If two or more wild-type cells acted in concert to induce centre formation the relationship would have been exponential, not linear.

The ten other aggregateless strains which were examined gave equivocal results. That is, the number of centres was not linearly proportional to the number of wild-type with which these mutants were paired. However, the centre: wild-type cell relationships showed a decreasing slope with increasing numbers of wild-type - the reverse of an exponential function. Secondly, the results obtained with mixtures of wild-type and mutants Agg-89 or -91 have shown that the linear relationship may hold only within a limited range of wild-type population sizes and be obscured outside of that range because of extraneous circumstances. Perhaps, therefore, a more detailed examination of the synergistic activities of the ten strains will reveal the existence of such limited ranges of proportionality. 
There is now a considerable body of evidence to suggest that the number of cells which can act as initiators depends markedly upon the nature of the audience which must be induced to aggregate. For the wild-type the proportion of initiators has been found to be either $1: 2200$ or $1: 76$, depending upon whether the audience consists of wild-type themselves at relatively low density $\left(200\right.$ cells $\left./ \mathrm{mm}^{2}{ }^{2}\right)$ or of mutant Agg-204 at very high density $\left(2 \times 10^{4}\right.$ cells $\left./ \mathrm{mm} .{ }^{2}\right)$. For the fruity mutant Fty-1, the ratio is either 1:24 or 1:63, depending on whether the fruity cells supply their own audience or whether the audience is made up of wild-type (Sussman, 1955).

At least two explanations may be invoked to account for these variations in initiator cell ratios, namely:

(1) There is a random distribution of initiative capacity in the population. A small proportion of cells possess high capacity; the remainder possess moderate or low capacities. In any system the number of cells which are potentially strong enough initiators actually to induce aggregation is determined by the innate sensitivity of the responder cells as well as by how closely the latter can be packed around the putative initators so as to receive the aggregative stimulus.

(2) The matrix of interactions within a population before the onset of aggregation can induce cells to become initiators; these then supply the aggregative stimulus. The higher the density at which the cells can be packed, the more intense can these interactions be and thus the greater the number of initiator cells. Wild-type cells can be packed only loosely because at densities above 200 cells $/ \mathrm{mm} .^{2}$ the initiator cells are already so close that they compete for the same audience and thus cannot make their presence known. But in synergistic mixtures, the wild-type is maintained at constant low density and it is only the aggregateless cells which are packed closely, thus permitting more intense interactions and the concommitant production of many more demonstrable initiators amongst the sparsely packed wild-type.

This second explanation has been given support by the experiments involving extracts of mutant and wild-type strains. However, they are not mutually exclusive and both may operate.

This investigation was supported by grants from the National Institutes of Health, U.S. Public Health Service, and from the Office of Naval Research.

Part of the experimental results reported here were taken from a thesis offered by the senior author (H. L. E.) in partial fulfilment of the requirements for the Ph.D. degree at Northwestern University.

The senior author was an N.S.F. pre-Doctoral Fellow during the course of this investigation. He is currently a post-Doctoral Fellow, National Institutes of Health, U.S. Public Health Service, at Northwestern University.

\section{REFERENCES}

Bonner, J.'T. (1947). Evidence for the formation of cell aggregation by chemotaxis in the development of the slime mold, Dictyostelium discoideum. J.exp. Zool.106, 1.

BonNer, J. T. \& Frascella, E. B. (1953). Variation in cell size during the development of the slime mold, Dictyostelium discoideum. Biol. Bull., Wood's Hole, 104, 297. 
Journal of General Microbiology, Vol. 18, No. 2

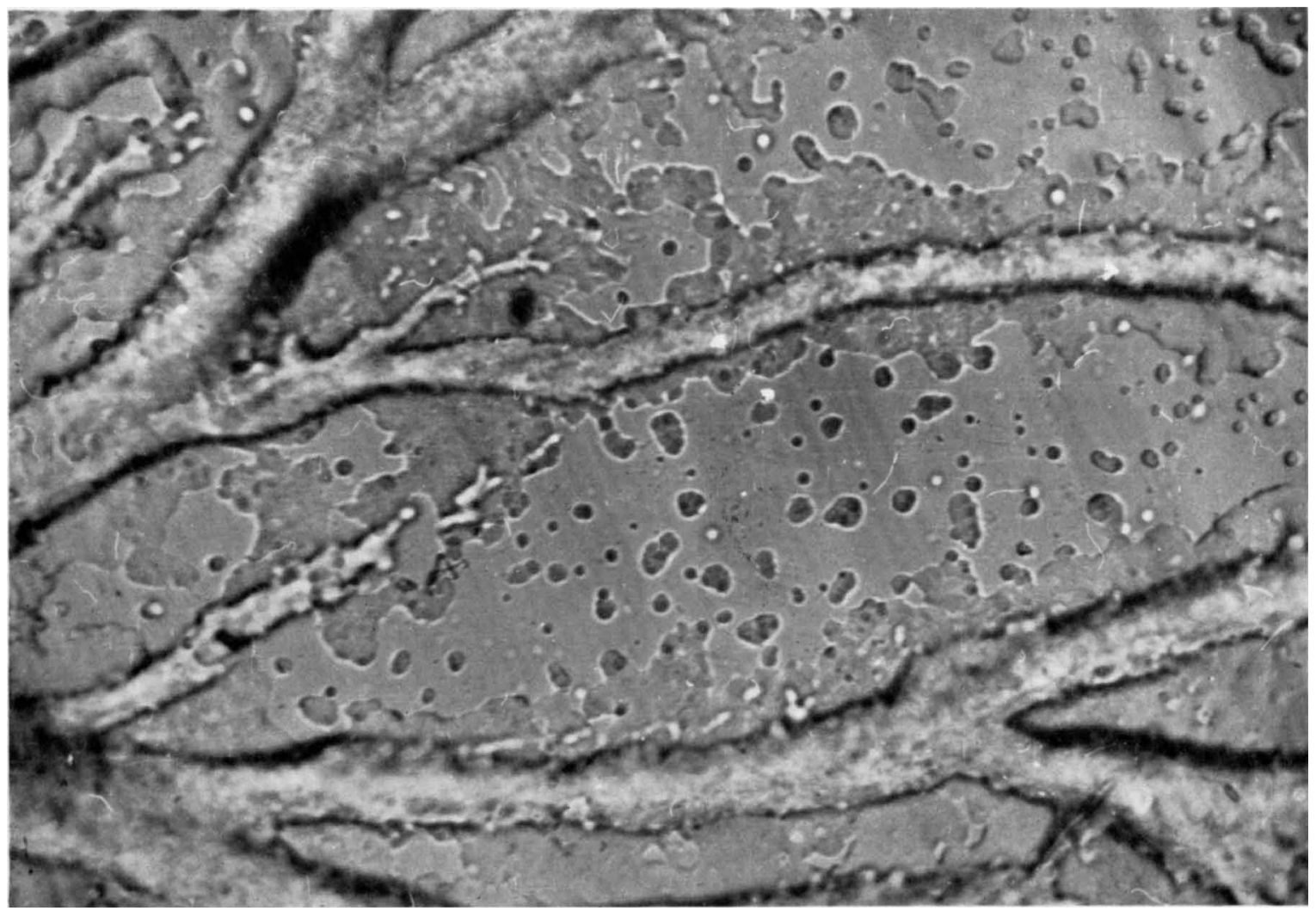

Fig. 1

H. L. Ennis and M. Sussman-Synergiste morplioglenesis. Plate 1

(Facing p. 448) 
Journal of General Microbiology, Vol. 18, No. 2

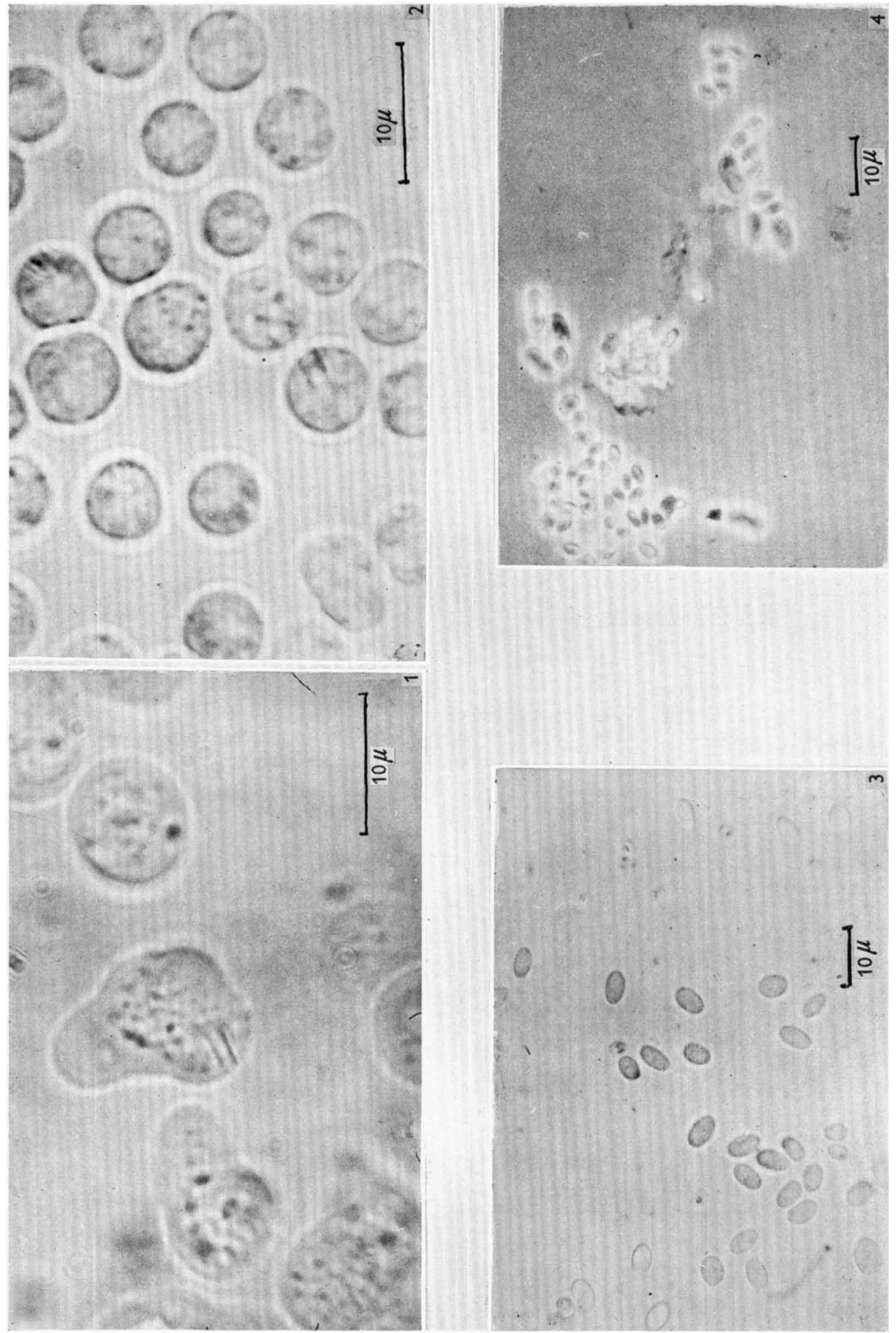

H. L. Ennis and M. Sussman-Synergistic morphogenesis. Plate 2 


\section{Synergistic morphogenesis}

Bonner, J. T. \& Slifkin, M. (1949). Study of the control of differentiation: proportions of spore and stalk cells in the slime mold, Dictyostelium discoideum. Amer. J. Bot. 36, 727.

Bradley, S. G., Sussman, M. \& Ennis, H. L. (1956). Environmental factors affecting the aggregation of the cellular slime mold, Dictyostelium discoideum. J. Protozool. 3, 33.

Davis, B. D. (1950). Studies on nutritionally deficient bacterial mutants isolated with penicillin. Experimentia, 6, 41 .

GrEGG, J. H. (1957). Serological investigations of aggregateless variants of the slime mold, Dictyostelium discoideum. Anat. Rec. 128, 558.

Grobstern, C. (1956). Inductive tissue interaction in development. Advanc. Cancer Res. 4, 221.

Sussman, M. (1951). Origin of cellular heterogeneity in the development of the slime molds, Dictyosteliaceae. J. exp. Zool. 118, 407.

Sussman, M. (1952). An analysis of the aggregation stage in the development of the slime molds, Dictyostelliaceae. II. Aggregative center formation by mixtures of Dictyostelium discoideum wild type and aggregateless variants. Biol. Bull., Woods Hole, 103, 446.

Sussman, M. (1954). Synergistic and antagonistic interactions between morphogenetically deficient variants of the slime mould, Dictyostelium discoideum. J. gen. Microbiol. 10, 110.

Sussman, M. (1955). 'Fruity' and other mutants of the cellular slime mould. Dictyostelium discoideum: a study of developmental aberrations. J. gen. Microbiol. 13, 295.

Sussman, M. (1956). On the relation between growth and morphogenesis in the slime mold, Dictyostelium discoideum. Biol. Bull., Woods Hole, $110,91$.

Sussman, M. \& LEE, F. (1955). Interactions among variant and wild type strains of cellular slime molds across thin agar membranes. Proc. nat. Acad. Sci., Wash. 41, 70.

Sussman, M. \& Noel, E. (1952). An analysis of the aggregation stage in the development of the slime molds, Dictyosteliaceae. I. The populational distribution of the capacity to initiate aggregation. Biol. Bull., Woods Hole, 103, 259.

Sussman, R. R. \& Sussman M. (1953). Cellular differentiation in Dictyosteliaceae: heritable modifications of the developmental pattern. Ann. N.Y. Acad. Sci. 56, 949, Art. 5.

\section{EXPLANATION OF PLATES}

\section{Plate 1}

1. Attraction of Dictyostelium discoideum mutant Agg-78 to wild-type aggregative streams across a thin agar membrane. The wild-type stream is well formed and tightly packed. The Agg-78 cells may be seen to cluster around these streams but do not produce wellformed streams of their own. Magnification, $\times \mathbf{8 6 0}$.

Plate 2

1. Dictyostelium discoideum wild-type vegetative myxamoebae.

2. Mutant Agg-91 vegetative dwarf myxamoebae. Note difference in size between the two strains.

3. Spores from wild-type fruiting bodies. Note the small variation in spore sizes in the sample.

4. Spores from fruiting bodies formed from a mixture of 500 wild-type and $6 \times 10^{5} \mathrm{Agg-91}$ cells plated in $0.01 \mathrm{ml}$. drops on washed agar. Note the great variation in size among the spores and the preponderance of small spores, presumably derived from Agg-91 dwarf myxamoebae. 